\title{
Article \\ Controllability for Retarded Semilinear Neutral Control Systems of Fractional Order in Hilbert Spaces
}

\author{
Daewook Kim ${ }^{1}(\mathbb{D})$ and Jin-Mun Jeong ${ }^{2, *(D)}$ \\ 1 Department of Mathematics Education, Seowon University, Cheongju 28674, Korea; kdw@seowon.ac.kr \\ 2 Department of Applied Mathematics, Pukyong National University, Busan 48513, Korea \\ * Correspondence: jmjeong@pknu.ac.kr
}

check for updates

Citation: Kim, D.; Jeong, J.-M. Controllability for Retarded Semilinear Neutral Control Systems of Fractional Order in Hilbert Spaces. Mathematics 2021, 9, 671. https:// doi.org/10.3390/math9060671

Academic Editor: Duarte Pedro Mata de Oliveira Valério

Received: 9 March 2021

Accepted: 19 March 2021

Published: 21 March 2021

Publisher's Note: MDPI stays neutral with regard to jurisdictional claims in published maps and institutional affiliations.

Copyright: (c) 2021 by the authors. Licensee MDPI, Basel, Switzerland. This article is an open access article distributed under the terms and conditions of the Creative Commons Attribution (CC BY) license (https:/ / creativecommons.org/licenses/by/ $4.0 /)$.

\begin{abstract}
In this paper, we discuss the approximate controllability for a class of retarded semilinear neutral control systems of fractional order by investigating the relations between the reachable set of the semilinear retarded neutral system of fractional order and that of its corresponding linear system. The research direction used here is to find the conditions for nonlinear terms so that controllability is maintained even in perturbations. Finally, we will show a simple example to which the main result can be applied.
\end{abstract}

Keywords: approximate controllability; fractional order differential equation; retarded neutral system; semilinear system

\section{Introduction}

Let $H$ and $V$ be two complex Hilbert spaces so that $V$ is a dense subspace of $H$. In this paper, we study the approximate controllability of the following semilinear retarded neutral functional differential control system of fractional order:

$$
\left\{\begin{array}{l}
\frac{d^{\alpha}}{d t^{\alpha}}\left[x(t)+g\left(t, x_{t}\right)\right]=A x(t)+\int_{-h}^{0} a(s) A_{1} x(t+s) d s+F(t, x(t))+B u(t), t>0, \\
x(0)=\phi^{0}, \quad x(s)=\phi^{1}(s), \quad-h \leq s<0,
\end{array}\right.
$$

where $h>0,1 / 2<\alpha<1,\left(\phi^{0}, \phi^{1}\right) \in H \times L^{2}(-h, 0 ; V), a(\cdot)$ is Hölder continuous, and $g, F$ are provided with functions that satisfy some assumptions described later. Moreover, $A$ is a densely defined closed linear operator that generates an analytic semigroup and $A_{1}$ is generally unbounded and closed linear operator that satisfies domain $D\left(A_{1}\right) \supset D(A)$. For a given $s \in[0, T], x_{s}:[-h, 0] \rightarrow H$ is defined as $x_{s}(r)=x(s+r)$ for $r \in[-h, 0]$. The controller $B$ is a bounded linear operator from $U$ to $H$, where $U$ is a Banach space of control variables.

This kind of systems occurs in many real practical mathematical models that arise in dynamic systems, science and engineering applications. For example, fractional differential equations are treated as another model of nonlinear differential equations [1-4], and the nonlinear vibrations of earthquake can be modeled as fractional derivatives [5]. There has been a significant development in applications and theory of fractional differential equations in recent years, see [6-9] and the references therein. Most of the work on neutral initial value problems dominated by delayed semilinear parabolic equation have been devoted to the existence of solutions and the control problems. Recently, the existence theory and applications for fractional neutral evolution equations has been devoted in $[7,10]$, and the existence and approximation of solutions to fractional evolution equation in Muslim [11]. In addition, Sukavanam et al. [12] have been studied approximate controllability of delayed fractional order semilinear equatins.

In this paper, we introduce a different approach from previous works. by assuming either the boundedness of an induced inverse of the controllability operator on quotient space as in [13] or the constraint of the Lipschitz constant of nonlinear term as in [12]. Our 
research direction is to take advantage of the regularity and variations of constant formula for solutions of the given systems using the fundamental solution that appears in a linear system. The result assert the equivalence condition between the controllability for the retarded neutral control system of fractional order and one for the associated the linear system excluded the nonlinear term.

The paper is organized as follows-in Section 2, we deal with the regularity and structure for solutions of semilinear fractional order retarded neutral functional differential equations and introduce basic properties. In Section 3, we will obtain the equivalent relations between the reachable set of the semilinear fractional order retarded neutral functional differential equation and that of its corresponding linear system. Finally, we will show a simple example to which the main result can be applied.

\section{Preliminaries and Lemmas}

\subsection{Retarded Linear Equations}

Let $V$ be a Hilbert space densely and continuously embedded in $H$. The norms of $V$ and $H$ are denoted by $\|\cdot\|$ and $|\cdot|$, respectively. The norm of dual space $V^{*}$ is denoted by $\|\cdot\|_{*}$. For simplicity, we can consider that

$$
\|u\|_{*} \leq|u| \leq\|u\|, \quad u \in V
$$

Let $b(\cdot, \cdot)$ be a bounded sesquilinear form defined in $V \times V$ satisfying Gårding's inequality:

$$
\operatorname{Re} b(u, u) \geq c_{0}\|u\|^{2}-c_{1}|u|^{2}, \quad c_{0}>0, \quad c_{1} \geq 0 .
$$

Let $A$ be the operator derived from the sesquilinear form $-b(\cdot, \cdot)$ :

$$
\left(\left(c_{1}-A\right) u, v\right)=-b(u, v), \quad u, v \in V,
$$

where $(\cdot, \cdot)$ denotes also the duality pairing between $V$ and $V^{*}$. From (2) it follows that for each $u \in V$

$$
\operatorname{Re}(A u, u) \geq c_{0}\|u\|^{2} \text {. }
$$

Thanks to the Lax-Milgram theorem, we know that $A$ is a bounded linear operator from $V$ to $V^{*}$. The realization of $A$ in $H$, which is the restriction of $A$ to domain

$$
D(A)=\{u \in V ; A u \in H\}
$$

with the graph norm

$$
\|u\|_{D(A)}=\left(|A u|^{2}+|u|^{2}\right)^{1 / 2}, \quad u \in D(A)
$$

is also denoted by $A$. It is proved in Theorem 3.6.1 of [14] that $A$ generates an analytic semigroup $S(t)=e^{t A}$ in both $H$ and $V^{*}$, and there exists a constant $M_{0}$ such that

$$
\|u\| \leq M_{0}\|u\|_{D(A)}^{1 / 2}|u|^{1 / 2}
$$

for every $u \in D(A)$. By identifying the dual of $H$ with $H$, we may consider the following sequence

$$
D(A) \subset V \subset H \subset V^{*} \subset D(A)^{*},
$$

where each space is continuous injection.

Lemma 1. In relation to (3), (4), we have

$$
\left(V, V^{*}\right)_{1 / 2,2}=H, \quad(D(A), H)_{1 / 2,2}=V,
$$

where $\left(V, V^{*}\right)_{1 / 2,2}$ denotes the real interpolation space between $V$ and $V^{*}$ (see Section 1.3 .3 of [15]). 
First, we consider the following linear time delayed functional differential equation with forcing term $k$ :

$$
\left\{\begin{array}{l}
x^{\prime}(t)=A x(t)+\int_{-h}^{0} a(s) A_{1} x(t+s) d s+k(t), \quad t>0, \\
x(0)=\phi^{0}, \quad x(s)=\phi^{1}(s) \quad-h \leq s \leq 0,
\end{array}\right.
$$

where $A_{1}$ is generally unbounded and closed linear operator that satisfies domain $D\left(A_{1}\right) \supset$ $D(A)$. In order to construct the fundamental solution of (5), we need to impose the following two condition:

Assumption 1. The function $a(\cdot)$ is assumed to be real valued and Hölder continuous of order $\rho$ in the interval $[-h, 0]$ :

$$
|a(s)| \leq H_{0}, \quad|a(s)-a(\tau)| \leq H_{0}(s-\tau)^{\rho}, \quad-h \leq \tau, s \leq 0
$$

for a constant $H_{0}$.

According to Nakaglri $[16,17]$, the fundamental solution $W(\cdot)$ to (5) is by definition a bounded the operator valued function satisfying

$$
\left\{\begin{array}{l}
W(t)=S(t)+\int_{0}^{t} S(t-s)\left\{\int_{-h}^{0} a(\tau) A_{1} W(s+\tau) d \tau\right\} d s, \quad t>0 \\
W(0)=I, \quad W(s)=0, \quad-h \leq s<0
\end{array}\right.
$$

where $S(\cdot)$ is the semigroup generated by $A$. For each $t>0$, we introduce the operator valued function $U_{t}(\cdot)$ defined by

$$
U_{t}(s)=\int_{-h}^{s} W(t-s+\sigma) a(\sigma) A_{1} d \sigma: V \rightarrow V, \quad s \in[-h, 0]
$$

Then (5) is represented

$$
\begin{aligned}
x(t) & =W(t) \phi^{0}+\int_{-h}^{0} U_{t}(s) \phi^{1}(s) d s+\int_{0}^{t} W(t-s) k(s) d s, \\
U_{t}(s) & =\int_{-h}^{s} W(t-s+\sigma) a(\sigma) A_{1} d \sigma .
\end{aligned}
$$

If $X$ and $Y$ are two Banach space, $\mathcal{L}(X, Y)$ is the collection of all bounded linear operators from $X$ into $Y$, and $\mathcal{L}(X, X)$ is simply written as $\mathcal{L}(X)$. From Proposition 4.1 of [18] or Theorem 1 of [19], it follows the following results.

Lemma 2. Under Assumption 1, the fundamental solution $W(t)$ to (5) exists uniquely and is bounded. Applying Proposition 4.1 of [18] to the Equation (5), there exists a constant $C_{0}>0$ such that

$$
\begin{aligned}
& \|W(t)\|_{\mathcal{L}(H)} \leq C_{0},\|W(t)\|_{\mathcal{L}\left(V^{*}, V\right)} \leq C_{0} / t \\
& \left\|W\left(t^{\prime}\right)-W(t)\right\|_{\mathcal{L}(H)} \leq C_{0}\left(t^{\prime}-t\right) \\
& \left\|W\left(t^{\prime}\right)-W(t)\right\|_{\mathcal{L}\left(V^{*}\right)} \leq C_{0}\left(t^{\prime}-t\right) \\
& \left\|W\left(t^{\prime}\right)-W(t)\right\|_{\mathcal{L}\left(V^{*}, V\right)} \leq C_{0}\left(t^{\prime}-t\right)^{\kappa}(t-h)^{-\kappa}
\end{aligned}
$$

for $h<t<t^{\prime}$, and $\kappa<\rho$. 


\subsection{Semilinear Fractional Order Differential Equations}

Consider the following retarded neutral differential system with fractional order:

$$
\left\{\begin{array}{l}
\frac{d^{\alpha}}{d t^{\alpha}}\left[x(t)+g\left(t, x_{t}\right)\right]=A x(t)+\int_{-h}^{0} a(s) A_{1} x(t+s) d s+F(t, x)+k(t), t>0, \\
x(0)=\phi^{0}, \quad x(s)=\phi^{1}(s), \quad-h \leq s<0,
\end{array}\right.
$$

where $0<\alpha<1, k$ is a forcing term, $A$ and $A_{1}$ are the linear operators defined as in Section 2.1. For each $s \in[0, T]$, we define $x_{s}:[-h, 0] \rightarrow H$ as

$$
x_{s}(r)=x(s+r), \quad-h \leq r \leq 0 .
$$

We will set

$$
\Pi=L^{2}(-h, 0 ; V) .
$$

Definition 1. Let $\Gamma$ be the Gamma function. The fractional integral of order $\alpha>0$ with the lower limit 0 from a function $f$ is defined by

$$
I^{\alpha} f(t)=\frac{1}{\Gamma(\alpha)} \int_{0}^{t} \frac{f(s)}{(t-s)^{1-\alpha}} d s, \quad t>0,
$$

provided the right hand side is pointwise defined on $[0, \infty)$.

The fractional derivative of order $\alpha>0$ in the Caputo sense with the lower limit 0 from a function $f \in C^{n}[0, \infty)$ is defined as

$$
\frac{d^{\alpha} f(t)}{d t^{\alpha}}=\frac{1}{\Gamma(n-\alpha)} \int_{0}^{t} \frac{f^{(n)}(s)}{(t-s)^{1+\alpha-n}} d s=I^{n-\alpha} f^{(n)}(t), \quad t>0, n-1<\alpha<n .
$$

We refer [20] for the fundamental consequences about fractional integrals and fractional derivative.

The mild solution of System (8) is represented as (see [10,21]):

$$
\begin{aligned}
& x(t)=S(t)\left[\phi^{0}+g\left(0, \phi^{1}\right)\right]-g\left(t, x_{t}\right)+\frac{1}{\Gamma(\alpha)} \int_{0}^{t}(t-s)^{(\alpha-1)} A S(t-s) g\left(s, x_{s}\right) d s \\
& +\frac{1}{\Gamma(\alpha)} \int_{0}^{t}(t-s)^{(\alpha-1)} S(t-s)\left\{\int_{-h}^{0} a_{1}(\tau) A_{1} x(s+\tau) d \tau+F(s, x(s))+k(s)\right\} d s .
\end{aligned}
$$

By using the fundamental solution $W(\cdot)$ described by (6) in the sense of Nakaglri [10], (8) is also represented by

$$
\begin{aligned}
x(t)= & W(t)\left[\phi^{0}+g\left(0, \phi^{1}\right)\right]+\int_{-h}^{0} U_{t}(s) \phi^{1}(s) d s-g\left(t, x_{t}\right) \\
& +\frac{1}{\Gamma(\alpha)} \int_{0}^{t}(t-s)^{(\alpha-1)} A W(t-s) g\left(s, x_{s}\right) d s \\
& +\frac{1}{\Gamma(\alpha)} \int_{0}^{t}(t-s)^{(\alpha-1)} W(t-s)\{F(s, x(s))+k(s)\} d s,
\end{aligned}
$$

where

$$
U_{t}(s)=\int_{-h}^{s} W(t-s+\sigma) a(\sigma) A_{1} d \sigma .
$$

Throughout this section, we assume $c_{1}=0$ in (2) to keep things simple without losing generality. So we have the closed half plane $\{\lambda: \operatorname{Re} \lambda \geq 0\} \subset \rho(A)$. Hence, it is possible to define the fractional power $A^{\alpha}$ for $\alpha>0$ so that the subspace $D\left(A^{\alpha}\right)$ with a norm

$$
\|x\|_{\alpha}=\left\|A^{\alpha} x\right\|, \quad x \in D\left(A^{\alpha}\right)
$$


is dense in $H$. It is also well known fact that $A^{\alpha}$ is a closed operator with its domain dense and $D\left(A^{\alpha}\right) \supset D\left(A^{\beta}\right)$ for $0<\alpha<\beta$. Due to the well known fact that $A^{-\alpha}$ is a bounded operator, there is a constant $C_{-\alpha}>0$ such that

$$
\left\|A^{-\alpha}\right\|_{\mathcal{L}(H)} \leq C_{-\alpha}, \quad\left\|A^{-\alpha}\right\|_{\mathcal{L}\left(V^{*}, V\right)} \leq C_{-\alpha}
$$

Lemma 3. For $t>0$, there is a positive constant $C_{\alpha}$ such that the following inequalities hold for all $t>0$ :

$$
\left\|A^{\alpha} W(t)\right\|_{\mathcal{L}(H)} \leq \frac{C_{\alpha}}{t^{\alpha}}, \quad\left\|A^{\alpha} W(t)\right\|_{\mathcal{L}(H, V)} \leq \frac{C_{\alpha}}{t^{3 \alpha / 2}}
$$

Proof. From ([14] [Lemma 3.6.2]), it follows that there exists a constant $C$ such that the following inequalities hold:

$$
\begin{aligned}
& \|S(t)\|_{\mathcal{L}\left(V^{*}, V\right)} \leq t^{-1} C \\
& \|A S(t)\|_{\mathcal{L}(H, V)} \leq t^{-3 / 2} C
\end{aligned}
$$

where $S(t)$ is an analytic semigroup generated by $A$. The relation (10) is immediately from the inequalities (11) and (12) by results of fractional power of $A$ and the definition of $W(t)$.

We need the following assumptions on System (8) to establish our results.

Assumption 2. Let $\mathcal{L}$ and $\mathcal{B}$ be the Lebesgue $\sigma$-field on $[0, \infty)$ and the Borel $\sigma$-field on $[-h, 0]$ respectively. Let $\mu$ be a Borel measure on $[-h, 0]$ and $f:[0, \infty) \times[-h, 0] \times V \times V \rightarrow H$ be a nonlinear mapping satisfying the following:

(i) For each $x, y \in V$, the mapping $f(\cdot, \cdot, x, y)$ is strongly $\mathcal{L} \times \mathcal{B}$-measurable;

(ii) There exist positive constants $L_{0}, L_{1}, L_{2}$ such that

$$
\left\{\begin{array}{l}
|f(t, s, x, y)-f(t, s, \hat{x}, \hat{y})| \leq K_{1}|| x-\hat{x}\left\|+K_{2}|| y-\hat{y}\right\| \\
|f(t, s, 0,0)| \leq K_{0}
\end{array}\right.
$$

for each $(t, s) \in[0, \infty) \times[-h, 0]$, and $x, \hat{x}, y, \hat{y} \in V$.

For $x \in L^{2}(-h, T ; V)$, Set

$$
F(t, x)=\int_{-h}^{0} f(t, s, x(t), x(t+s)) \mu(d s) .
$$

Here, the operator $F$ is the nonlinear part of quasilinear equations as seen in Yong and Pan [22].

Assumption 3. Let $g:[0, T] \times \Pi \rightarrow H$ be a nonlinear mapping so that there is a $L_{g}>0$ satisfying the following conditions:

(i) For each $x \in \Pi$, the mapping $g(\cdot, x)$ is strongly measurable;

(ii) There is a positive constant $\beta>1-2 \alpha / 3$ such that

$$
\left|A^{\beta} g(t, 0)\right| \leq L_{g}, \quad\left|A^{\beta} g(t, x)-A^{\beta} g(s, \hat{x})\right| \leq L_{g}\left(|t-s|+|| x-\hat{x} \|_{\Pi}\right),
$$

for all $s, t \in[0, T]$, and $x, \hat{x} \in \Pi$.

Lemma 4. Let $x \in L^{2}(-h, T ; V), T>0$. Then $F(\cdot, x) \in L^{2}(0, T ; H)$. and

$$
\begin{aligned}
\|F(\cdot, x)\|_{L^{2}(0, T ; H)} \leq & \mu([-h, 0])\left\{K_{0} \sqrt{T}+\left(K_{1}+K_{2}\right)\|x\|_{L^{2}(0, T ; V)}\right. \\
& \left.+K_{2}\|x\|_{L^{2}(-h, 0 ; V)}\right\}
\end{aligned}
$$


Moreover, if $x_{1}, x_{2} \in L^{2}(-h, T ; V)$, then

$$
\begin{aligned}
& \left\|F\left(\cdot, x_{1}\right)-F\left(\cdot, x_{2}\right)\right\|_{L^{2}(0, T ; H)} \leq \mu([-h, 0]) \\
& \quad \times\left\{\left(K_{1}+K_{2}\right)\left\|x_{1}-x_{2}\right\|_{L^{2}(0, T ; V)}+K_{2}\left\|x_{1}-x_{2}\right\|_{L^{2}(-h, 0 ; V)}\right\} .
\end{aligned}
$$

Proof. From Assumption 2, it is easily seen that

$$
\begin{aligned}
& \|F(\cdot, x)\|_{L^{2}(0, T ; H)} \leq \mu([-h, 0])\left\{K_{0} \sqrt{T}+K_{1}\|x\|_{L^{2}(0, T ; V)}+K_{2}\|x\|_{L^{2}(-h, T ; V)}\right\} \\
& \quad \leq \mu([-h, 0])\left\{K_{0} \sqrt{T}+\left(K_{1}+K_{2}\right)\|x\|_{L^{2}(0, T ; V)}+K_{2}\|x\|_{L^{2}(-h, 0 ; V)}\right\} .
\end{aligned}
$$

The proof of (13) is the same argument.

For each $s \in[0, T]$, we define $x_{s}:[-h, 0] \rightarrow H$ as

$$
x_{s}(r)=x(s+r), \quad-h \leq r \leq 0 .
$$

Lemma 5. Suppose that $x_{s}(r)=x(s+r)(-h \leq r \leq 0)$ for $s \in[0, T]$. Then the mapping $s \mapsto x_{s}$ belongs to $C([0, T] ; \Pi)$, and

$$
\begin{aligned}
& \left\|x_{t}\right\|_{\Pi} \leq\|x\|_{L^{2}(-h, t ; V)}(0<t<T), \\
& \|x .\|_{L^{2}(0, T ; \Pi)} \leq \sqrt{T}\|x\|_{L^{2}(-h, T ; V)} .
\end{aligned}
$$

Proof. The first inequality (14) is easy to verify. Moreover, since

$$
\left\|x_{t}\right\|_{\Pi}=\left[\int_{-h}^{0}\|x(s+\tau)\|^{2} d \tau\right]^{1 / 2} \leq\left[\int_{-h}^{t}\|x(\tau)\|^{2} d \tau\right]^{1 / 2} \leq\|x\|_{L^{2}(-h, t ; V)}, t>0,
$$

and

$$
\begin{aligned}
\|x .\|_{L^{2}(0, T ; \Pi)}^{2} & \leq \int_{0}^{T}\left\|x_{s}\right\|_{\Pi}^{2} d s \leq \int_{0}^{T} \int_{-h}^{0}\|x(s+r)\|^{2} d r d s \\
& \leq \int_{0}^{T} d s \int_{-h}^{T}\|x(r)\|^{2} d r \leq T\|x\|_{L^{2}(-h, T ; V)}^{2}
\end{aligned}
$$

the proof of (15) is completed.

Remark 1. Here, we note that by using interpolation theory, we have

$$
\begin{aligned}
& L^{2}(0, T ; D(A)) \cap W^{1,2}(0, T ; H) \subset C([0, T] ; V), \\
& L^{2}(0, T ; V) \cap W^{1,2}\left(0, T ; V^{*}\right) \subset C([0, T] ; H) .
\end{aligned}
$$

Thus, there exists a constant $C_{1}>0$ such that

$$
\begin{aligned}
& \|x\|_{C([0, T] ; V)} \leq C_{1}\|x\|_{L^{2}(0, T ; D(A)) \cap W^{1,2}(0, T ; H)}, \\
& \|x\|_{C([0, T] ; H)} \leq C_{1}\|x\|_{L^{2}(0, T ; V) \cap W^{1,2}\left(0, T ; V^{*}\right)} .
\end{aligned}
$$

By virtue of Theorem 2.1 of [23], we have the following result on the corresponding linear equation of (8).

Proposition 1. Suppose that Assumptions 1-3 be satisfied. Then for $\left(\phi^{0}, \phi^{1}\right) \in H \times \Pi$ and $k \in L^{2}\left(0, T ; V^{*}\right)$, there is a solution $x$ of System (8) such that

$$
x \in L^{2}(0, T ; V) \cap W^{1,2}\left(0, T ; V^{*}\right) \hookrightarrow C([0, T] ; H),
$$


and there is a constant $C_{2}$ such that

$$
\begin{aligned}
& \|x\|_{L^{2}(-h, T ; V)} \leq C_{2}\left(1+\left|\phi^{0}\right|+\left\|\phi^{1}\right\|_{\Pi}+\|k\|_{L^{2}\left(0, T ; V^{*}\right)}\right), \\
& \left\|x_{t}-x_{s}\right\|_{\Pi} \leq|t-s|^{\hat{\eta}} C_{2}\left(1+\left|\phi^{0}\right|+\left\|\phi^{1}\right\|_{\Pi}+\|\left. k\right|_{L^{2}\left(0, T ; V^{*}\right)}\right),
\end{aligned}
$$

where

$$
|t-s|^{\eta}:=\max \left\{|t-s|^{1 / 2},|t-s|^{(2 \alpha+3 \beta-2) / 2}\right\} .
$$

Using Lemma 1 we can follow the argument of Proposition 1 term by term to deduce the following result.

Proposition 2. Suppose that Assumptions 1-3 be satisfied. Then for $\left(\phi^{0}, \phi^{1}\right) \in H \times L^{2}(-h, 0 ; D(A))$ and $k \in L^{2}(0, T ; H)$, there is a solution $x$ of System (8) such that

$$
x \in L^{2}(0, T ; D(A)) \cap W^{1,2}(0, T ; H) \hookrightarrow C([0, T] ; V),
$$

and there is a constant $C_{2}$ such that

$$
\|x\|_{L^{2}(0, T ; D(A)) \cap W^{1,2}(0, T ; H)} \leq C_{2}\left(1+\left\|\phi^{0}\right\|+\left\|\phi^{1}\right\|_{L^{2}(-h, 0 ; D(A))}+\|k\|_{L^{2}(0, T ; H)}\right) .
$$

\section{Approximate Reachable Sets}

Let $U$ is a Banach space of control variables and let the controller $B$ be a bounded linear operator from $U$ to $H$. In this section, we concern with the approximate controllability of the following semilinear neutral control system with delays of fractional order:

$$
\left\{\begin{array}{l}
\frac{d^{\alpha}}{d t^{\alpha}}\left[x(t)+g\left(t, x_{t}\right)\right]=A x(t)+\int_{-h}^{0} a(s) A_{1} x(t+s) d s+F(t, x(t))+B u(t), \\
x(0)=0, \quad x(s)=0, \quad-h \leq s<0 .
\end{array}\right.
$$

The solution $x(t)=x(t ; g, F, u)$ of $(3,1)$ is the following form:

$$
\begin{aligned}
x(t)= & -g\left(t, x_{t}\right)+\frac{1}{\Gamma(\alpha)} \int_{0}^{t}(t-s)^{(\alpha-1)} A W(t-s) g\left(s, x_{s}\right) d s \\
& +\frac{1}{\Gamma(\alpha)} \int_{0}^{t}(t-s)^{(\alpha-1)} W(t-s)\{F(s, x(s))+B u(s)\} d s .
\end{aligned}
$$

Noting that

$$
x(T ; 0,0, u)=\frac{1}{\Gamma(\alpha)} \int_{0}^{t}(t-s)^{(\alpha-1)} W(t-s) B u(s) d s,
$$

for $T>0, \phi \in H \times \Pi$ and $u \in L^{2}(0, T ; U)$, we define reachable sets as follows.

$$
\begin{aligned}
& L_{T}(\phi)=\left\{x(T ; 0,0, u): u \in L^{2}(0, T ; U)\right\}, \\
& R_{T}(\phi)=\left\{x(T ; g, F, u): u \in L^{2}(0, T ; U)\right\}, \\
& L(\phi)=\bigcup_{T>0} L_{T}(\phi), \quad R(\phi)=\bigcup_{T>0} R_{T}(\phi) .
\end{aligned}
$$

Definition 2. (1) System (19) is said to be H-approximately controllable for the initial value $\phi$ (resp. in time T) if $\overline{R(\phi)}=H\left(\right.$ resp. $\left.\overline{R_{T}(\phi)}=H\right)$.

(2) The linear system corresponding (19) is said to be H-approximately controllable for the initial value $\phi($ resp. in time $T)$ if $\overline{L(\phi)}=H\left(\right.$ resp. $\left.\overline{L_{T}(\phi)}=H\right)$.

Remark 2. Since A generate an analytic semigroup, the following (1)-(4) are equivalent for the linear system (see ([24] [Theorem 3.10])).

(1) $\overline{L(\phi)}=H \quad \forall \phi \in H \times \Pi$. 
(2) $\overline{L(0)}=H$.

(3) $\overline{L_{T}(\phi)}=H \quad \forall \phi \in H \times \Pi$.

(4) $\overline{L_{T}(0)}=H$.

Theorem 1. Let Assumptions 1-3 hold. If $\alpha>1 / 2$ then we have

$$
\overline{R_{T}(0)} \subset \overline{L_{T}(0)}, \quad T>0 .
$$

Proof. Let $z_{0} \notin \overline{L_{T}(0)}$. Since $\overline{L_{T}(0)}$ is a balanced closed convex subspace, we have $\rho z_{0} \notin$ $\overline{L_{T}(0)}$ for every $\rho \in \mathbb{R}$, and

$$
\inf \left\{\left|z_{0}-z\right|: z \in \overline{L_{T}(0)}\right\}=d,
$$

and

By the Formula (17) we have

$$
\left.\left\|\left.x\right|_{L^{2}(0, T ; V)}:=\right\| x(\cdot ; 0, F, u)\right|_{L^{2}(0, T ; V)} \leq C_{2}\left(1+\|B\|_{\mathcal{L}(H, U)} \mid\|u\|_{L^{2}(0, T ; U)}\right),
$$

where $C_{2}$ is the constant in Proposition 1. Put

$$
\begin{aligned}
N:= & \left(C_{-\beta}+\frac{T^{\alpha+\beta-1}}{(\alpha+\beta-1) \Gamma(\alpha)} C_{1-\beta}\right) L_{g}\left(\|x\|_{L^{2}(0, T ; V)}+1\right) \\
& +C_{1} C_{2}\left[1+\mu([-h, 0])\left\{K_{0} \sqrt{T}+\left(K_{1}+K_{2}\right) C_{2}\left(1+\|B\|_{\mathcal{L}(H, U)}\|u\|_{L^{2}(0, T ; U)}\right\}\right],\right.
\end{aligned}
$$

where $C_{1-\beta}, C_{1}$ and $C_{2}$ are constant of (10), (16) and (17), respectively. For every $u \in$ $L^{2}(0, T ; U)$, we choose a constant $\rho>0$ such that

$$
N<\rho d .
$$

By virtue of Assumption 3, we deduce that

$$
\begin{aligned}
\left|g\left(t, x_{t}\right)\right| & =\left|A^{-\beta} A^{\beta} g\left(t, x_{t}\right)\right| \leq C_{-\beta} L_{g}\left(\left\|x_{t}\right\|_{\Pi}+1\right) \\
& \leq C_{-\beta} L_{g}\left(\|x\|_{L^{2}(0, T ; V)}+1\right) .
\end{aligned}
$$

By (10), we have

$$
\begin{aligned}
\left|(t-s)^{(\alpha-1)} A S(t-s) g\left(s, x_{s}\right)\right| & =(t-s)^{(\alpha-1)}|| A^{1-\beta} S(t-s)||_{\mathcal{L}(H)} \mid A^{\beta}\left(g\left(s, x_{s}\right) \mid\right. \\
& \leq \frac{C_{1-\beta}}{(t-s)^{1-\alpha+(1-\beta)}} \mid A^{\beta}\left(g\left(s, x_{s}\right) \mid\right. \\
& \leq \frac{C_{1-\beta}}{(t-s)^{2-(\alpha+\beta)}} L_{g}\left(\|\left. x\right|_{L^{2}(0, T ; V)}+1\right),
\end{aligned}
$$

so that

$$
\begin{aligned}
& \left|\frac{1}{\Gamma(\alpha)} \int_{0}^{t}(t-s)^{(\alpha-1)} A W(t-s) g\left(s, x_{s}\right) d s\right| \\
& \leq \frac{1}{\Gamma(\alpha)} C_{1-\beta} L_{g}\left(\|x\|_{L^{2}(0, T ; V)}+1\right) \int_{0}^{t} \frac{1}{(t-s)^{2-(\alpha+\beta)}} d s \\
& \leq \frac{T^{\alpha+\beta-1}}{(\alpha+\beta-1) \Gamma(\alpha)} C_{1-\beta} L_{g}\left(\|x\|_{L^{2}(0, T ; V)}+1\right) .
\end{aligned}
$$


Moreover, by Hölder inequality and Lemma 5,

$$
\begin{aligned}
& \left|\frac{1}{\Gamma(\alpha)} \int_{0}^{t}(t-s)^{(\alpha-1)} W(t-s) F(s, x(s)) d s\right| \\
& \left.\leq \frac{C_{0} T^{\alpha-1 / 2}}{\sqrt{2 \alpha-1} \Gamma(\alpha)}\|F(\cdot, x)\|_{L^{2}(0, T ; H)}\right) \\
& \leq \frac{C_{0} T^{\alpha-1 / 2}}{\sqrt{2 \alpha-1} \Gamma(\alpha)} \mu([-h, 0])\left\{K_{0} \sqrt{T}+\left(K_{1}+K_{2}\right)\|x\|_{L^{2}(0, T ; V)}\right\} \\
& \leq \frac{C_{0} T^{\alpha-1 / 2}}{\sqrt{2 \alpha-1} \Gamma(\alpha)} \mu([-h, 0])\left\{K_{0} \sqrt{T}+\left(K_{1}+K_{2}\right) C_{2}\left(1+\|B\|_{\mathcal{L}(H, U)}\|u\|_{L^{2}(0, T ; U)}\right)\right\} .
\end{aligned}
$$

Thus, form (20) and (21), it follows that

$$
\begin{aligned}
& \left|x(T ; g, f, u)-\rho z_{0}\right| \\
& \geq\left|\frac{1}{\Gamma(\alpha)} \int_{0}^{t}(t-s)^{(\alpha-1)} W(t-s) B u(s) d s-\rho z_{0}\right| \\
& \quad-\left|-g\left(t, x_{t}\right)+\frac{1}{\Gamma(\alpha)} \int_{0}^{t}(t-s)^{(\alpha-1)}\left\{A W(t-s) g\left(s, x_{s}\right)+W(t-s) F(s, x(s))\right\} d s\right| \\
& \geq \rho d-N>0 .
\end{aligned}
$$

Thus, we have $\rho z_{0} \notin \overline{R_{T}(0)}$.

Lemma 6. For $0 \leq \tau<t \leq T$, let $\mathcal{B}(\tau, t)$ from $L^{2}(0, T ; H)$ into $H$ be defined by

$$
\mathcal{B}(\tau, t) k:=\frac{1}{\Gamma(\alpha)} \int_{\tau}^{t}(t-s)^{\alpha-1} W(t-s) k(s) d s .
$$

Then we have

$$
|\mathcal{B}(\tau, t) k| \leq\left.\frac{C_{0}(t-\tau)^{\alpha-1 / 2}}{\Gamma(\alpha) \sqrt{2 \alpha-1}}|| k\right|_{L^{2}(0, T ; H)}:=|t-\tau|^{\alpha-1 / 2} L_{B}|| k||_{L^{2}(0, T ; H)} .
$$

Proof. The proof is immediately obtained by a simple calculation and using Hölder inequality.

Thanks for Lemma 6, we assume that there exists a positive constant $L_{B}$ such that

$$
\|\hat{\mathcal{B}}(s, t)\|_{\mathcal{L}\left(L^{2}(0, T ; H), H\right)} \leq|t-s|^{\alpha-1 / 2} L_{B} .
$$

Theorem 2. Let Assumptions 1-3 hold. If $\alpha>1 / 2$ then we have

$$
{\overline{L_{T}(0)}}^{V} \subset \overline{R_{T}(0)}, \quad T>0,
$$

where ${\overline{L_{T}(0)}}^{V}$ is the closure of $L_{T}(0)$ in $V$. Therefore, if the linear system (19) with $g \equiv 0$ and $F \equiv 0$ is $V$-approximately controllable, then the nonlinear system (19) is also H-approximately controllable.

Proof. Let $\gamma>0$ be arbitrary given. We will show that $z \in{\overline{L_{T}(0)}}^{V}$ satisfying $\|z\|<\gamma$ belongs to $\overline{R_{T}(0)}$. Let $u \in L^{2}(0, T ; U)$ be arbitrary fixed. Then by (17) we have

$$
\left\|x_{u}\right\|_{L^{2}(0, T ; V)} \leq C_{2}\left(1+\|B\|_{\mathcal{L}(H, U)}\|u\|_{L^{2}(0, T ; U)}\right),
$$


where $x_{u}$ is the solution (19) corresponding to the control $u$. For any $\epsilon>0$, we can choose positive constants $\delta$ and $\eta$ satisfying

$$
\begin{aligned}
& \delta^{\eta}:=\max \{\left.\delta, \delta^{\alpha-1 / 2}, \delta^{\alpha+\beta-1}\right\} \\
&<\min \left\{\frac{10}{\epsilon}, \quad\left[C_{0} C_{-\beta} L_{g}\left(T+\|x\|_{L^{2}(0, T ; V)}+1\right)\right.\right. \\
&+C_{-\beta} L_{g}+C_{2}\left(1+\|B\|_{\mathcal{L}(H, U)}\|u\|_{L^{2}(0, T ; U)}\right) \\
&\left.\quad+\left\{\gamma+C_{1} C_{2}\left(1+C_{0}\|B\|_{\mathcal{L}(H, U)}\|u\|_{L^{2}(0, T ; U)}\right)\right\}\right]^{-1} \\
& {\left[\frac{T^{\alpha+\beta-1} C_{0} L_{g}+1}{(\alpha+\beta-1) \Gamma(\alpha)} C_{1-\beta}\left(\left\|x_{u}\right\|_{L^{2}(0, T ; V)}+1\right)\right]^{-1} } \\
& \\
&\left(M_{0}+\frac{\epsilon \hat{M}_{0}}{10}\right)^{-1}, \\
& {\left[( L _ { B } + \frac { 1 } { \sqrt { 2 \alpha - 1 } \Gamma ( \alpha ) } ) \left\{C _ { 0 } \mu ( [ - h , 0 ] ) \left\{K_{0} \sqrt{T}\right.\right.\right.} \\
&\left.\left.+\left.\left(K_{1}+K_{2}\right)\left\|x_{u}\right\|\right|_{L^{2}(0, T ; V)}\right\}\right]^{-1}, \\
& \\
&\left(M_{1}+\frac{\epsilon \hat{M}_{2}}{10}\right)^{-1}, \\
& {\left.\left[\left(C_{0}+1\right) C_{0} \sqrt{T}\|B\|\|u\|_{L^{2}(0, T ; U)}\right]^{-1}\right\} \epsilon / 10, }
\end{aligned}
$$

where

$$
\begin{aligned}
M_{0}= & \frac{\delta^{\alpha+\beta-1}}{(\alpha+\beta-1) \Gamma(\alpha)}\left\{C_{2}\left(1+\|B\|_{\mathcal{L}(H, U)}\|u\|_{L^{2}(0, T ; U)}\right)\right. \\
& +\left(\gamma+C_{1} C_{2}\left(1+C_{0}\|B\|_{\mathcal{L}(H, U)}\|u\|_{L^{2}(0, T ; U)}\right)+1\right\}, \\
\hat{M}_{0}= & \frac{\delta^{\alpha+\beta-1}}{(\alpha+\beta-1) \Gamma(\alpha)} \\
M_{1}= & L_{B} \mu([-h, 0])\left[K_{0} \sqrt{T}+\left(K_{1}+K_{2}\right)\left\{C_{2}\left(1+\|B\|\left\|_{\mathcal{L}(H, U)}\right\| u \|_{L^{2}(0, T ; U)}\right)\right.\right. \\
& \left.+\left(\gamma+C_{1} C_{2}\left(1+C_{0}\|B\|\left\|_{\mathcal{L}(H, U)}\right\| u \|_{L^{2}(0, T ; U)}\right)\right\}\right], \\
\hat{M}_{1}= & L_{B} \mu([-h, 0])\left(K_{1}+K_{2}\right) .
\end{aligned}
$$

Set

$$
\begin{aligned}
x_{1}:= & x(T-\delta ; g, F, u) \\
= & -g\left(T-\delta,\left(x_{u}\right)_{T-\delta}\right) \\
& +\frac{1}{\Gamma(\alpha)} \int_{0}^{T-\delta}(T-\delta-s)^{\alpha-1} A W(T-\delta-s) g\left(s,\left(x_{u}\right)_{s}\right) d s \\
& +\frac{1}{\Gamma(\alpha)} \int_{0}^{T-\delta}(T-\delta-s)^{\alpha-1} W(T-\delta-s) F\left(s, x_{u}(s)\right) d s+\mathcal{B}(0, T-\delta) u,
\end{aligned}
$$

where $x_{u}(t)=x(t ; g, F, u)$ for $0<t \leq T$. Consider the following linear problem:

$$
\left\{\begin{array}{l}
\frac{d^{\alpha}}{d t^{\alpha}} y(t)=A_{0} y(t)+\int_{-h}^{0} a(s) A_{1} y(t+s) d s+B u(t), t>0, \\
y(T-\delta)=x_{1}, \quad y(s)=0 \quad-h \leq s \leq 0 .
\end{array}\right.
$$


The solution of (26) with respect to a control $w \in L^{2}(T-\delta, T ; U)$ is denoted by

$$
\begin{aligned}
y_{w}(T)= & W(\delta) x_{1}+\mathcal{B}(T-\delta, T) w \\
= & -W(\delta) g\left(T-\delta,\left(x_{u}\right)_{T-\delta}\right) \\
& +\frac{1}{\Gamma(\alpha)} W(\delta) \int_{0}^{T-\delta}(T-\delta-s)^{\alpha-1} A W(T-\delta-s) g\left(s,\left(x_{u}\right)_{s}\right) d s \\
& +\frac{1}{\Gamma(\alpha)} W(\delta) \int_{0}^{T-\delta}(T-\delta-s)^{\alpha-1} W(T-\delta-s) F\left(s, x_{u}(s)\right) d s \\
& +W(\delta) \mathcal{B}(0, T-\delta) u+\mathcal{B}(T-\delta, T) w .
\end{aligned}
$$

By Remark 2, we know that $\overline{L_{T}(\phi)}=\overline{L_{T}(0)}=\overline{L(0)}$ is independent of the time $T$ for any initial data $\phi \in H \times \Pi$. Hence, since $z \in{\overline{L_{T}(0)}}^{V}$, there exists $w_{1} \in L^{2}(T-\delta, T ; U)$ such that

$$
\left\|y_{w_{1}}(T)-z\right\|<\frac{\epsilon}{10}
$$

Now we set

$$
v(s)= \begin{cases}u & \text { if } 0 \leq s \leq T-\delta \\ w_{1}(s) & \text { if } T-\delta<s<T\end{cases}
$$

Then $v \in L^{2}(0, T ; U)$. Observing that

$$
\begin{aligned}
x_{v}(t ; g, F, v) & =-g\left(t,\left(x_{v}\right)_{t}\right)+\frac{1}{\Gamma(\alpha)} \int_{0}^{t}(t-s)^{\alpha-1} A W(t-s) g\left(s,\left(x_{v}\right)_{s}\right) d s \\
& +\frac{1}{\Gamma(\alpha)} \int_{0}^{t}(t-s)^{\alpha-1} W(t-s)\left\{F\left(s, x_{v}(s)+B v(s)\right\} d s,\right.
\end{aligned}
$$

from (27) and (28), we have

$$
\begin{aligned}
\left|x_{v}(T ; g, F, v)-z\right| \leq & \left|y_{w_{1}}(T)-z\right| \\
+ & \left|-W(\delta) g\left(T-\delta,\left(x_{u}\right)_{T-\delta}\right)+g\left(T,\left(x_{v}\right)_{T}\right)\right| \\
& +\frac{1}{\Gamma(\alpha)} \mid \int_{0}^{T}(T-s)^{\alpha-1} A W(T-s) g\left(s,\left(x_{v}\right)_{s}\right) d s \\
& -W(\delta) \int_{0}^{T-\delta}(T-\delta-s)^{\alpha-1} A W(T-\delta-s) g\left(s,\left(x_{u}\right)_{s}\right) d s \mid \\
+ & \frac{1}{\Gamma(\alpha)} \mid \int_{0}^{T}(T-s)^{\alpha-1} W(T-s) F\left(s, x_{v}(s)\right) d s \\
& -W(\delta) \int_{0}^{T-\delta}(T-\delta-s)^{\alpha-1} W(T-\delta-s) F\left(s, x_{u}(s)\right) d s \mid \\
+ & \frac{1}{\Gamma(\alpha)} \mid \int_{0}^{T-\delta}(T-s)^{\alpha-1} W(T-s) B u(s) d s \\
& -W(\delta) \int_{0}^{T-\delta}(T-\delta-s)^{\alpha-1} W(T-\delta-s) B v(s) \mid \\
\leq & \frac{\epsilon}{10}+I+I I+I I I+I V+V .
\end{aligned}
$$

First, let us begin to evaluate the term of I. From (7), (9), (14) and Assumption 3, it follows that

$$
\begin{aligned}
\left|(W(\delta)-1) g\left(T-\delta,\left(x_{u}\right)_{T-\delta}\right)\right| & \leq \delta C_{0}\left|A^{-\beta} A^{\beta} g\left(T-\delta,\left(x_{u}\right)_{T-\delta}\right)\right| \\
& =\delta C_{0} C_{-\beta} L_{g}\left(|T-\delta|+\left\|\left(x_{u}\right)_{t}\right\|_{\Pi}+1\right) \\
& \leq \delta C_{0} C_{-\beta} L_{g}\left(|T-\delta|+\|x\|_{L^{2}(0, T ; V)}+1\right),
\end{aligned}
$$


and

$$
\left|g\left(T-\delta,\left(x_{u}\right)_{T-\delta}\right)-g\left(T,\left(x_{u}\right)_{T-\delta}\right)\right| \leq \delta C_{-\beta} L_{g} .
$$

By (14) and (18), we have

$$
\begin{aligned}
& \left.\left|g\left(T,\left(x_{u}\right)_{T-\delta}\right)-g\left(T,\left(x_{v}\right)_{T}\right)\right| \leq L_{g} \|\left(x_{u}\right)_{T-\delta}\right)-\left(x_{v}\right)_{T} \|_{\Pi} \\
& \leq \delta^{\hat{\eta}} L_{g} C_{2}\left(1+\|B\|_{\mathcal{L}(H, U)}|| u \|_{L^{2}(0, T ; U)}\right)+\left\|\mathcal{B}(T-\delta, T) w_{1}\right\|_{L^{2}(T-\delta, T ; V)},
\end{aligned}
$$

where $\delta^{\eta}$ is the constant of (18). Now we will protest to calculate the estimate of $\| \mathcal{B}(T-$ $\delta, T) w_{1} \|_{L^{2}(T-\delta, T ; V)}$. By (27), we have

$$
\left\|\mathcal{B}(T-\delta, T) w_{1}\right\| \leq\left\|y_{w_{1}}(T)-z\right\|+\gamma+\left\|W(\delta) x_{1}\right\| \leq \frac{\epsilon}{10}+\gamma+\left\|W(\delta) x_{1}\right\| .
$$

Here, consider the following problem:

$$
\left\{\begin{array}{l}
\frac{d^{\alpha}}{d t^{\alpha}}\left[\hat{x}(t)+\hat{g}\left(t, \hat{x}_{t}\right)\right]=A \hat{x}(t)+\int_{-h}^{0} a(s) A_{1} \hat{x}(t+s) d s+\hat{F}(t, \hat{x}(t))+\hat{B} u(t), t>0, \\
\hat{x}(0)=0, \quad \hat{x}(s)=0, \quad-h \leq s<0,
\end{array}\right.
$$

where

$$
\hat{g}\left(t, \hat{x}_{t}\right)=W(\delta) g\left(t, \hat{x}_{t}\right), \quad \hat{F}(t, \hat{x}(t))=W(\delta) F(t, \hat{x}(t)), \quad \text { and } \hat{B}=W(\delta) B .
$$

Then $\hat{g}\left(t, \hat{x}_{t}\right), \hat{F}(t, \hat{x}(t))$ and $\hat{B}$ are satisfied the conditions of Proposition 2. Since the solution $\hat{x}$ of (30) on $[0, T-\delta]$ is represented by $W(\delta) x_{1}$ in the sense of (26), it follows from (16), (30) and Proposition 2 that

$$
\begin{aligned}
\left\|W(\delta) x_{1}\right\| & \leq C_{1}\left\|W(\delta) x_{1}\right\|_{\mathcal{W}_{0}(T-\delta)} \leq C_{1} C_{2}\left(1+\|\hat{B} u\|_{L^{2}(0, T ; H)}\right) \\
& \leq C_{1} C_{2}\left(1+C_{0}\|B\|_{\mathcal{L}(H, U)}\|u\|_{L^{2}(0, T ; U)}\right)
\end{aligned}
$$

which implies

$$
\left\|\mathcal{B}(T-\delta, T) w_{1}\right\|_{L^{2}(0, T ; V)} \leq \sqrt{\delta}\left(\gamma+\frac{\epsilon}{10}+C_{1} C_{2}\left(1+C_{0}\|B\|_{\mathcal{L}(H, U)}\|u\|_{L^{2}(0, T ; U)}\right) .\right.
$$

Hence, (32) and (34) implies

$$
\begin{aligned}
\left|g\left(T,\left(x_{u}\right)_{T-\delta}\right)-g\left(T,\left(x_{v}\right)_{T}\right)\right| \leq & \delta^{\hat{\eta}} L_{g} C_{2}\left(1+\left.\|B\|\right|_{\mathcal{L}(H, U)} \mid\|u\|_{L^{2}(0, T ; U)}\right) \\
& +\sqrt{\delta}\left\{\gamma+\frac{\epsilon}{10}+C_{1} C_{2}\left(1+C_{0}|| B\left\|_{\mathcal{L}(H, U)}\right\| u \|_{L^{2}(0, T ; U)}\right)\right\} .
\end{aligned}
$$

From (30), (31), (35), and Assumption 3, it follows that

$$
\begin{aligned}
I= & \left|W(\delta) g\left(T-\delta,\left(x_{u}\right)_{T-\delta}\right)-g\left(T,\left(x_{v}\right)_{T}\right)\right| \\
\leq & \left|(W(\delta)-I) g\left(T-\delta,\left(x_{u}\right)_{T-\delta}\right)\right|+\left|g\left(T-\delta,\left(x_{u}\right)_{T-\delta}\right)-g\left(T,\left(x_{u}\right)_{T-\delta}\right)\right| \\
& +\left|g\left(T,\left(x_{u}\right)_{T-\delta}\right)-g\left(T,\left(x_{v}\right)_{T}\right)\right| \\
\leq & \delta C_{0} C_{-\beta} L_{g}\left(|T-\delta|+|| x \|_{L^{2}(0, T ; V)}+1\right)+\delta C_{-\beta} L_{g} \\
& +\delta^{\hat{\eta}} C_{2}\left(1+\|B\|_{\mathcal{L}(H, U)}|| u \|_{L^{2}(0, T ; U)}\right) \\
& +\sqrt{\delta}\left\{\gamma+\frac{\epsilon}{10}+C_{1} C_{2}\left(1+C_{0}\|B\|_{\mathcal{L}(H, U)}\|u\|_{L^{2}(0, T ; U)}\right)\right\} .
\end{aligned}
$$


By (17) and (34), we get

$$
\begin{aligned}
\left\|x_{w_{1}}\right\|_{L^{2}(T-\delta, T ; V)} \leq & \left\|x_{\mathcal{v}}\right\|_{L^{2}(0, T ; V)} \\
\leq & \left\|x_{\mathcal{v}}\right\|_{L^{2}(0, T-\delta ; V)}+\left\|\mathcal{B}(T-\delta, T) w_{1}\right\|_{L^{2}(0, T ; V)} \\
\leq & C_{2}\left(1+\|B\|\left\|_{\mathcal{L}(H, U)}\right\| u \|_{L^{2}(0, T ; U)}\right) \\
& +\sqrt{\delta}\left\{\gamma+\frac{\epsilon}{10}+C_{1} C_{2}\left(1+C_{0}\|B\|_{\mathcal{L}(H, U)}\|u\|_{L^{2}(0, T ; U)}\right\},\right.
\end{aligned}
$$

and with aid of Lemma 3 and Assumption 3, we have

$$
\begin{aligned}
& \left|(t-s)^{\alpha-1} A W(t-s) g\left(s, x_{s}\right)\right| \\
& =\left.(t-s)^{\alpha-1}|| A^{1-\beta} W(t-s)\right|_{\mathcal{L}(H)} \mid A^{\beta}\left(g\left(s, x_{s}\right) \mid\right. \\
& \leq \frac{C_{1-\beta}}{(t-s)^{2-\alpha-\beta}} \mid A^{\beta}\left(g\left(s, x_{s}\right) \mid \leq \frac{C_{1-\beta}}{(t-s)^{2-\alpha-\beta}} L_{g}\left(\|x\|_{L^{2}(0, T ; V)}+1\right) .\right.
\end{aligned}
$$

Hence, from (37) and (38), it follows that

$$
\begin{aligned}
& \frac{1}{\Gamma(\alpha)}\left|\int_{T-\delta}^{T}(T-s)^{\alpha-1} A W(T-s) g\left(s, x_{w_{1}}(s)\right) d s\right| \\
& \leq \frac{\delta^{\alpha+\beta-1}}{(\alpha+\beta-1) \Gamma(\alpha)}\left(|| x_{w_{1}} \|_{L^{2}(0, T ; V)}+1\right) \leq \delta^{\alpha+\beta-1}\left(M_{0}+\frac{\epsilon \hat{M}_{0}}{10}\right),
\end{aligned}
$$

where $M_{0}$ and $\hat{M}_{0}$ are the constants of (22) and (23), respectively. By (39), Assumption 3 and Lemma 2,

$$
\begin{aligned}
& I I=\mid \frac{1}{\Gamma(\alpha)} \int_{0}^{T}(T-s)^{\alpha-1} A W(T-s) g\left(s,\left(x_{v}\right)_{s}\right) d s \\
& -\frac{1}{\Gamma(\alpha)} W(\delta) \int_{0}^{T-\delta}(T-\delta-s)^{\alpha-1} A W(T-\delta-s) g\left(s,\left(x_{u}\right)_{s}\right) d s \mid \\
& \leq \frac{1}{\Gamma(\alpha)} \mid I-(W(\delta)) \int_{0}^{T-\delta}(T-\delta-s)^{\alpha-1}\left\{A W(T-\delta-s) g\left(s, x_{u}(s)\right) d s \mid\right. \\
& +\frac{1}{\Gamma(\alpha)}\left|\int_{0}^{T-\delta}\left\{(T-s)^{\alpha-1}-(T-\delta-s)^{\alpha-1}\right\} A W(T-s) g\left(s,\left(x_{u}\right)_{s}\right) d s\right| \\
& +\frac{1}{\Gamma(\alpha)}\left|\int_{T-\delta}^{T}(T-s)^{\alpha-1} A W(T-s) g\left(s, x_{w_{1}}(s)\right) d s\right| \\
& \leq \frac{\delta T^{\alpha+\beta-1} C_{0} L_{g}+\delta^{\alpha+\beta-1}}{(\alpha+\beta-1) \Gamma(\alpha)} C_{1-\beta}\left(|| x_{u}||_{L^{2}(0, T ; V)}+1\right) \\
& +\delta^{\alpha+\beta-1}\left(M_{0}+\frac{\epsilon \hat{M}_{0}}{10}\right)<\frac{\epsilon}{5} .
\end{aligned}
$$

Hence, with the aid of Lemma 5, (37) and by using Hölder inequality, we have

$$
\begin{aligned}
& \frac{1}{\Gamma(\alpha)}\left|\int_{T-\delta}^{T}(T-s)^{\alpha-1} W(T-s) F\left(s, x_{w_{1}}(s)\right) d s\right| \\
& \leq \delta^{\alpha-1 / 2} L_{B}|| F\left(\cdot, x_{w_{1}}\right) \|_{L^{2}(0, T ; H)} \\
& \leq \delta^{\alpha-1 / 2} L_{B} \mu([-h, 0])\left\{K_{0} \sqrt{T}+\left(K_{1}+K_{2}\right)|| x_{w_{1}} \|_{L^{2}(T-\delta, T ; V)}\right\} \\
& \leq \delta^{\alpha-1 / 2}\left(M_{1}+\frac{\epsilon \hat{M}_{1}}{10}\right),
\end{aligned}
$$


where $M_{1}$ and $\hat{M}_{1}$ are the constants of (24) and (25), respectively. Hence, by virtue of Lemma 5 and using Hölder inequality, we have

$$
\begin{aligned}
& I I I=\mid \frac{1}{\Gamma(\alpha)} \int_{0}^{T}(T-s)^{\alpha-1} W(T-s) F\left(s, x_{v}(s)\right) d s \\
& -\frac{1}{\Gamma(\alpha)} W(\delta) \int_{0}^{T-\delta}(T-\delta-s)^{\alpha-1} W(T-\delta-s) F\left(s, x_{u}(s)\right) d s \mid \\
& \leq \frac{1}{\Gamma(\alpha)} \mid I-(W(\delta)) \int_{0}^{T-\delta}(T-\delta-s)^{\alpha-1}\left\{W(T-\delta-s) F\left(s, x_{u}(s)\right) d s \mid\right. \\
& +\frac{1}{\Gamma(\alpha)}\left|\int_{0}^{T-\delta}\left\{(T-s)^{\alpha-1}-(T-\delta-s)^{\alpha-1}\right\} W(T-s) F\left(s, x_{v}(s)\right) d s\right| \\
& +\frac{1}{\Gamma(\alpha)}\left|\int_{T-\delta}^{T}(T-s)^{\alpha-1} W(T-s) F\left(s, x_{w_{1}}(s)\right) d s\right| \\
& \leq\left(\delta L_{B}+\frac{\delta^{\alpha-1 / 2}}{\sqrt{2 \alpha-1} \Gamma(\alpha)}\right)\left\{C _ { 0 } \mu ( [ - h , 0 ] ) \left\{K_{0} \sqrt{T}\right.\right. \\
& \left.+\left(K_{1}+K_{2}\right)|| x_{u} \|_{L^{2}(0, T ; V)}\right\}+\delta^{\alpha-1 / 2}\left(M_{1}+\frac{\epsilon \hat{M}_{1}}{10}\right)<\frac{\epsilon}{5^{\prime}}
\end{aligned}
$$

and

$$
\begin{aligned}
I V= & \left|W(\delta) \int_{0}^{T-\delta} W(T-\delta-s) B u(s) d s-\int_{0}^{T-\delta} W(T-s) B u(s) d s\right| \\
\leq & \left|(W(\delta)-I) \int_{0}^{T-\delta} W(T-\delta-s) B u(s) d s\right| \\
& +\left|\int_{0}^{T-\delta}(W(T-\delta-s)-W(T-s)) B u(s) d s\right| \\
\leq & \left(C_{0}+1\right) C_{0} \delta \sqrt{T}|| B \|\left._{\mathcal{L}(H, U)}|| u\right|_{L^{2}(0, T ; U)}<\frac{\epsilon}{10} .
\end{aligned}
$$

Therefore, by (29) and (36)-(43), we have

$$
\|x(T ; \phi, G, v)-z\|<\epsilon,
$$

that is, $z \in \overline{R_{T}(\phi)}$ and the proof is complete.

Noting that ${\overline{L_{T}(0)}}^{V}=\overline{L_{T}(0)} \cap V$ and $V$ is dense in $H$, from Theorems 1 and 2, we obtain the following control results of (19).

Corollary 1. Under Assumptions 1-3, we have

$$
\overline{L_{T}(\phi)}=H \Longleftrightarrow \overline{R_{T}(\phi)}=H, \quad T>0 .
$$

Therefore, the H-approximate controllability of linear system (19) with $g \equiv 0$ and $F \equiv 0$ is equivalent to the condition for the H-approximate controllability of the nonlinear system (19).

\section{Example}

Let

$$
H=L^{2}(0, \pi), V=H_{0}^{1}(0, \pi), V^{*}=H^{-1}(0, \pi),
$$


and let $U$ be a Banach space of control variables. Consider the following retarded neutral differential system of fractional order in Hilbert space $H$ :

$$
\left\{\begin{array}{c}
\frac{d^{\alpha}}{d t^{\alpha}}\left[x(t, y)+g\left(t, x_{t}(t, y)\right)\right]=A x(t, y)+\int_{-h}^{0} a_{1}(s) A_{1} x(t+s, y) d s \\
+F(t, x(t))++B u(t, y), \quad(t, y) \in[0, T] \times[0, \pi] \\
x(0, y)=\phi^{0}(y), \quad x(s, y)=\phi^{1}(s, y), \quad(s, y) \in[-h, 0) \times[0, \pi],
\end{array}\right.
$$

where $h>0, a_{1}(\cdot)$ is Hölder continuous, $B \in \mathcal{L}(U, H)$, and $A_{1} \in \mathcal{L}(H)$. Let

$$
a(u, v)=\int_{0}^{\pi} \frac{d u(y)}{d y} \frac{\overline{d v(y)}}{d y} d y .
$$

Then

$$
A=\partial^{2} / \partial y^{2} \quad \text { with } \quad D(A)=\left\{x \in H^{2}(0, \pi): x(0)=x(\pi)=0\right\} .
$$

The eigenvalue and the eigenfunction of $A$ are $\lambda_{n}=-n^{2}$ and $z_{n}(y)=(2 / \pi)^{1 / 2} \sin n y$, respectively. Moreover,

(a1) $\left\{z_{n}: n \in N\right\}$ is an orthogonal basis of $H$ and

$$
S(t) x=\sum_{n=1}^{\infty} e^{n^{2} t}\left(x, z_{n}\right) z_{n}, \quad \forall x \in H, t>0 .
$$

Moreover, there exists a constant $M_{0}$ such that $\|S(t)\|_{\mathcal{L}(H)} \leq M_{0}$.

(a2) Let $0<\alpha<1$. Then the fractional power $A^{\alpha}: D\left(A^{\alpha}\right) \subset H \rightarrow H$ of $A$ is given by

$$
A^{\alpha} x=\sum_{n=1}^{\infty} n^{2 \alpha}\left(x, z_{n}\right) z_{n}, D\left(A^{\alpha}\right):=\left\{x: A^{\alpha} x \in H\right\} .
$$

In particular,

$$
A^{-1 / 2} x=\sum_{n=1}^{\infty} \frac{1}{n}\left(x, z_{n}\right) z_{n}, \text { and }\left\|A^{-1 / 2}\right\|=1 .
$$

The nonlinear mapping $F$ is the nonlinear part of quasilinear equations considered by Yong and Pan [22]. Define $g:[0, T] \times \Pi \rightarrow H$ as

$$
g\left(t, x_{t}\right)=\sum_{n=1}^{\infty} \int_{0}^{t} e^{n^{2} t}\left(\int_{-h}^{0} a_{2}(s) x(t+s) d s, z_{n}\right) z_{n}, \quad, t>0 .
$$

Then it can be checked that Assumption 3 is satisfied. Indeed, for $x \in \Pi$, we know

$$
A g\left(t, x_{t}\right)=(S(t)-I) \int_{-h}^{0} a_{2}(s) x(t+s) d s,
$$

where $I$ is the identity operator form $H$ to itself and

$$
\left|a_{2}(0)\right| \leq H_{2}, \quad\left|a_{2}(s)-a_{2}(\tau)\right| \leq H_{2}(s-\tau)^{\kappa}, \quad s, \tau \in[-h, 0]
$$

for a constant $\kappa>0$. Hence we have

$$
\begin{aligned}
\left|A g\left(t, x_{t}\right)\right| & \leq\left(M_{0}+1\right)\left\{\left|\int_{-h}^{0}\left(a_{2}(s)-a_{2}(0)\right) x(t+s) d \tau\right|+\left|\int_{-h}^{0} a_{2}(0) x(t+s) d \tau\right|\right\} \\
& \leq\left(M_{0}+1\right) H_{2}\left\{(2 \kappa+1)^{-1} h^{2 \rho+1}+h\right\}|| x_{t} \|_{\Pi} .
\end{aligned}
$$

It is immediately seen that Assumption 3 has been satisfied. Thus, all the conditions stated in Theorems 1 and 2 have been satisfied for the Equation (44). Therefore, 
by Theorems 1 and 2, we get that the approximate controllability of the general retarded linear differential system corresponding to (44) with $g \equiv 0$ and $F \equiv 0$ is equivalent to the condition for the $H$-approximate controllability of the semilinear system (44) for any $\alpha>\frac{1}{2}$.

\section{Conclusions}

This paper deals with the approximate controllability for a class of retarded semilinear neutral control systems of fractional order by investigating the relations between the reachable set of the semilinear retarded neutral system of fractional order and that of its corresponding linear system. The research direction used here is to find more general hypotheses of nonlinear terms so that controllability is maintained even in perturbations. The technique used here is to take advantage of the regularity and basic properties for solutions of the given systems using the fundamental solution that appears in a linear system. The result assert the equivalence condition between the controllability for the retarded neutral control system of fractional order and one for the associated the linear system excluded the nonlinear term, which can be also applied to the functional analysis concerning nonlinear control problems.

Author Contributions: Conceptualization, D.K. and J.-M.J. All authors have made equal contributions. All authors read and approved the final manuscript.

Funding: This research received no external funding.

Acknowledgments: Authors are thankful to the anonymous referee for useful comments and suggestions, which really helped us to improve our old manuscript. This work was supported by the National Research Foundation of Korea(NRF) grant funded by the Korea government(MSIT) (2019R1F1A1048077).

Conflicts of Interest: The authors declare that they have no competing interests.

\section{References}

1. Bonilla, B.; Rivero, M.; Rodriruez-Germa, L.; Trujillo, J.J. Fractional differential equations as alternative models to nonlinear differential equations. Appl. Math. Comput. 2007, 187, 79-88. [CrossRef]

2. Lakshmikantham, V.; Leela, S.; Vasundhara Devi, J. Theory of Fractional Dynamic Systems; Cambridge Scientific Publishers Ltd.: Cambridge, UK, 2009.

3. Miller, K.S.; Ross, B. An Introduction to the Fractional Calculus and Differential Equations; John Wiley: New York, NY, USA, 1993.

4. Kang, Y.H.; Jeong, J.M. Control problems for semi-linear retarded integro-differential equations by the Fredholm theoty. Int. J. Control 2019, 92, 56-64. [CrossRef]

5. Delbosco, D.; Rodino, L. Existence and uniqueness for a nonlinear fractional differential equation. J. Math. Anal. Appl. 1996, 204, 609-625. [CrossRef]

6. Hilfer, R. Applications of Fractional Calculus in Physics; World Scientific: Singapore, 2000.

7. Kilbas, A.A.; Srivastava, H.M.; Juan Trujillo, J. Theory and Applications of Fractional Differential Equations in: North-Holland Mathematics Studies 204; Elsevier Science B.V: Amsterdam, The Netherlands, 2006.

8. Ganesh, G.; Sakthivel, R.; Ren, Y.; Anthoni, S.M.; Mahmudov, N.I. Controllability of Neutral Fractional Functional Equations with Impulses and Infinite Delay. Abstr. Appl. Anal. 2013, 2013, 1-12. [CrossRef]

9. Dabas, J.; Chauhan, A. Existence and uniqueness of mild solution for an impulsive neutral fractional integro-differential equation with infinite delay. Math. Comput. Model. 2013, 57, 754-763. [CrossRef]

10. Jardat, O.K.; Al-Omari, A.; Momani, S. Existence of the mild solution for fractional semilinear initial value problems. Nonlinear Anal. 2008, 69, 3153-3159. [CrossRef]

11. Muslim, M. Existence and approximation of solutions to fractional differential equations. Math. Comput. Model. 2009, 49, 1164-1172. [CrossRef]

12. Sukavanam, N.; Kumar, S. Approximate controllability of fractional order semilinear with delay systems. J. Optim. Theory Appl. 2011, 151, 373-384. [CrossRef]

13. Balachadran, P.; Park, J.Y. Controllability of fractional integrodifferential systems with in Banach spaces. Nonlinear Anal. Hybrid Syst. 2009, 3, 363-367. [CrossRef]

14. Tanabe, H. Equations of Evolution; Pitman-London: London, UK, 1979.

15. Triebel, H. Interpolation Theory, Function Spaces, Differential Operators; North-Holland Publication: Amsterdam, The Netherlands, 1978.

16. Nakagiri, S. Optimal control of linear retarded systems in Banach spaces. J. Math. Anal. Appl. 1986, 120, 169-210. [CrossRef]

17. Nakagiri, S. Structural properties of functional differential equations in Banach spaces. Osaka J. Math. 1988, 25, 353-398. 
18. Jeong, J.M. Stabilizability of retarded functional differential equation in Hilbert space. Osaka J. Math. 1991, 28, 347-365.

19. Tanabe, H. Fundamental solutions for linear retarded functional differential equations in Banach space. Funkcial. Ekvac. 1992, 35, 149-177.

20. Podlubny, I. Fractional Differential Equations; Academic Press: San Diego, CA, USA, 1999.

21. Zhou, Y.; Jiao, F. Existence of mild solutions for fractional neutral evolution equations. Comput. Math. Appl. 2010, 59, 1063-1077. [CrossRef]

22. Yong, J.; Pan, L. Quasi-linear parabolic partial differential equations with delays in the highest order partial derivatives. J. Aust. Math. Soc. 1993, 54, 174-203. [CrossRef]

23. Cho, S.H.; Jeong, J.M.; Kang, Y.H. Regularity for fractional order retarded neutral differential equations in Hilbert spaces. J. Korean Math. Soc. 2016, 53, 1019-1036 [CrossRef]

24. Fattorini, H.O. Boundary control systems. SIAM J. Control Optim. 1968, 6, 349-402. [CrossRef] 